\title{
Wearable Technology: Trends and Opportunities for Organizations
}

\author{
Karen Robson, Leyland Pitt, Jan Kietzmann, Wade Halvorson, \\ and Asa Wallstrom
}

\begin{abstract}
This special session intends to explore the use of wearable technology in greater detail. Current perspectives identify a number of fundamental questions regarding wearable technology. Wearable technology is emerging as one of the key areas companies are investing in. In March 2014, Facebook announced that it had plans to acquire Oculus VR, a firm that produces virtual reality headsets. In May 2014, Apple announced its $\$ 3.2$ billion purchase of Beats Electronics. Within the next 3 years, wearable technology is predicted to be worth $\$ 10$ billion, with 170 million wearable technological devices in general use.

Currently, the biggest area for wearables is sports and fitness. For example, Nike's Fitbit exercise tracking and monitoring device. Another area in which wearables are growing in importance is in healthcare. A new Google project involves contact lenses for diabetics that will give the wearer a continuous readout on blood glucose levels. This will make a continuous physical testing procedure such as finger pricking or urine testing redundant. Another device, the Owlet (an ankle strap) will continuously monitor critical health indicators in newborns. The manufacturers of this device want to put it on every newborn, among others to monitor physical warnings of SIDS (sudden infant death syndrome) and infant sleep apnea.
\end{abstract}

\footnotetext{
K. Robson $(\bowtie) \bullet$ L. Pitt $\bullet$ J. Kietzmann

Simon Fraser University, Burnaby, BC, Canada

e-mail: krobson@sfu.ca; 1pitt@sfu.cal; lpitt@sfu.ca

W. Halvorson

SP Jain School of Management, Singapore, Singapore

e-mail: wade.halvorson@spjain.org
}

\author{
A. Wallstrom \\ Lulea University of Technology, Lulea, Sweden \\ e-mail: Asa.Wallstrom@ltu.se
}

